Journal of Mathematics and Statistics 5 (4): 408-410, 2009

ISSN 1549-3644

(C) 2009 Science Publications

\title{
Elementary Combinatorial-Probabilistic Proof of the Wallis and Stirling Formulas
}

\author{
Mikhail Kovalyov \\ Department of Mathematical and Statistical Sciences, \\ University of Alberta, Edmonton, Alberta, Canada T6G 2E1
}

\begin{abstract}
This short note provides a remarkably simple derivation of the Wallis and Stirling formulas based on elementary estimates applied to binomial coefficients. As a byproduct an elementary proof of the central limit theorem for the binomial distribution is also obtained.
\end{abstract}

Key words: Wallis formula, Stirling formula, normal distribution

\section{INTRODUCTION}

The Wallis formula ${ }^{[1,2,6]}$ :

$$
\lim _{n \rightarrow+\infty} \frac{2^{2 n}(n !)^{2}}{(2 n) ! \sqrt{n}}=\sqrt{\pi}
$$

and closely related Stirling formula:

$$
\lim _{m \rightarrow+\infty} \frac{2^{m} m !}{m^{m} \sqrt{m}}=\sqrt{2 \pi}
$$

are ubiquitous in Mathematics and its applications. Yet of many proofs available in the literature none is quite simple or "elementary". Attempts to find a simple and elementary proof have not stopped to this day as manifested by regular papers appearing in the literature, some of the recent and not so recent ones are given in Bibliography ${ }^{[1-6]}$. What constitutes a simple and/or elementary proof is, of course, in the eyes of the beholder-what is simple to one may be fairly complicated to another. The simplest proof known to the author is in ${ }^{[5]}$; yet it does not appear to be natural in the sense that it is a proof to an already known result without revealing how one may arrive at the result. Somewhat more natural is the probabilistic proof in $^{[3]}$; yet it is fairly complicated.

Here we provide a derivation of the Wallis formula by examining the binomial probability distribution, the simplest probability distribution known. The Stirling formula trivially follows from the Wallis formula.

Consider a one-dimensional random walk of a point $\mathrm{Q}$ that starts at $\mathrm{x}=0$ and at each step jumps from the point it occupies to either right or left adjacent integer with probability $1 / 2$ :

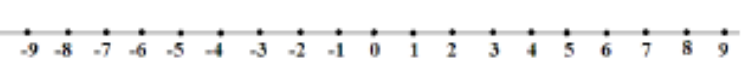

The probability of finding point $\mathrm{P}$ in location $\mathrm{x}=\mathrm{K}$ after $\mathrm{N}$ steps from its initial position is given by the binomial distribution:

$\mathrm{P}(\mathrm{K}, \mathrm{N})$

$=\left\{\begin{array}{l}\frac{\mathrm{N} !}{2^{\mathrm{N}}\left(\frac{\mathrm{N}+\mathrm{K}}{2} !\right)\left(\frac{\mathrm{N}-\mathrm{K}}{2} !\right)}, \text { if }|\mathrm{K}| \leq \mathrm{N} ; \mathrm{N}-\mathrm{K} \text { is even, } \\ 0, \text { otherwise }\end{array}\right.$

Let us now assume that:

$\mathrm{N}=2 \mathrm{n}$ is even

and $\varepsilon$ satisfies:

$0<\varepsilon<\frac{5}{30}$

Formula (3) can be rewritten as:

$\mathrm{P}(-2 \mathrm{~K}, 2 \mathrm{n})=\mathrm{P}(2 \mathrm{~K}, 2 \mathrm{n})$

$$
=\left\{\begin{array}{l}
\frac{(2 n) !}{2^{2 n}(n !)^{2}} \cdot \frac{(n !)^{2}}{(n+k) !(n-k) !}= \\
\frac{(2 n) !}{2^{2 n}(n !)^{2}} \prod_{j=1}^{k} \frac{n-(k-j)}{n+j}, \text { if } 0 \leq k \leq n \\
0, \quad \text { otherwise }
\end{array}\right.
$$

Formula (5) implies that for sufficiently large n:

$1-\sum_{|\mathrm{k}| \mathrm{n}^{0.5+\varepsilon}} \mathrm{P}(2 \mathrm{k}, 2 \mathrm{n})=\sum_{|\mathrm{k}| \geq \mathrm{n}^{0.5+\varepsilon}} \mathrm{P}(2 \mathrm{k}, 2 \mathrm{n}) \leq 2^{-\mathrm{n}^{2 \varepsilon}}$ 
and for $|\mathrm{k}| \leq \mathrm{n}^{0.5+\varepsilon}$ :

$$
\frac{1}{\sqrt{n}} \mathrm{e}^{-\frac{k^{2}}{n}-8 n^{-0.538}} \leq \frac{2^{2 n}(n !)^{2}}{(2 n) ! \sqrt{n}} P(2 k, 2 n) \leq \frac{1}{\sqrt{n}} e^{-\frac{k 2}{n}+8 n^{-0.5+3 e}}
$$

The proof of formulas (6) and (7) is given in the Appendix.

Formula (7) implies:

$$
\begin{aligned}
& {\left[\frac{1}{\sqrt{n}} \sum_{|k| \leq n^{0.5+\varepsilon}} e^{-\frac{k^{2}}{n}}\right] e^{-8 n^{-0.5+38}} \leq \frac{2^{2 n}(n !)^{2}}{(2 n) ! \sqrt{n}} \sum_{|k| \leq n^{0.5+\varepsilon}} P(2 k, 2 n)} \\
& \leq\left[\frac{1}{\sqrt{n}} \sum_{|k| \leq n^{0.5+\varepsilon}} e^{-\frac{k^{2}}{n}}\right] e^{-8 n^{-0.5+38}}
\end{aligned}
$$

Taking now limit as $\mathrm{n} \rightarrow+\infty$ and using $\lim _{n \rightarrow+\infty} \mathrm{e}^{8 \mathrm{n}^{-0.5+3 e}}=\lim _{\mathrm{n} \rightarrow+\infty} \mathrm{e}^{-8 \mathrm{n}^{-0.5+3 e}}=1, \lim _{\mathrm{n} \rightarrow+\infty} \sum_{|\mathrm{k}| \mathrm{n}^{0.5+8}} \mathrm{P}(2 \mathrm{k}, 2 \mathrm{n})=1$ due to (6), $\lim _{N \rightarrow+\infty} \frac{1}{\sqrt{n}} \sum_{\substack{k<\leq n^{05+e} \\ \text { kiseven }}} \mathrm{e}^{-\frac{k^{2}}{n}}=\int_{-\infty}^{+\infty} \mathrm{e}^{-t^{2}} \mathrm{dt}=\sqrt{\pi}$ we obtain (1).

What formulas (6 and 7) tell us is that inside $|\mathrm{k}| \leq \mathrm{n}^{0.5+\varepsilon}$ probability $\mathrm{P}(2 \mathrm{k} ; 2 \mathrm{n}) \approx \frac{1}{\sqrt{\pi \mathrm{n}}} \mathrm{e}^{-\frac{\mathrm{k} 2}{\mathrm{n}}}$ while the total probability outside of $|\mathrm{k}| \leq \mathrm{n}^{0.5+\varepsilon}$ is negligibly small.

To obtain (2) consider sequence $f_{m}=\frac{e^{m} m !}{m^{m} \sqrt{m}}$. Since $\mathrm{f}_{\mathrm{m}}>0, \frac{\mathrm{f}_{\mathrm{m}+1}}{\mathrm{f}_{\mathrm{m}}}=\frac{\mathrm{e}}{\left(1+\frac{1}{\mathrm{~m}}\right)^{\mathrm{m}+0.5}}<1, \mathrm{f}_{\mathrm{m}}$ is positive and monotonically decreasing from $\mathrm{f}_{1}=\mathrm{e}$ and hence must have a limit $\lim _{\mathrm{m} \rightarrow+\infty} f_{m}=A \geq 0$. Since due to (1) $\left(\lim _{\mathrm{m} \rightarrow+\infty} \mathrm{f}_{\mathrm{m}}\right)=\sqrt{2 \pi} \lim _{\mathrm{m} \rightarrow+\infty} \mathrm{f}_{2 \mathrm{~m}}$, limit $\lim _{\mathrm{m} \rightarrow+\infty} \mathrm{f}_{\mathrm{m}}$ must be either 0 or $\sqrt{2 \pi}$; the former is easily ruled out leaving us with the latter.

Note that besides the formula $\int_{-\infty}^{+\infty} \mathrm{e}^{-t^{2}} \mathrm{dt}=\sqrt{\pi}$ which requires the knowledge of double integrals usually covered in the second year of non-honors Calculus, the derivations provided here do not require any knowledge beyond the high school non-honors Calculus and basic probability/combinatorics.

Appendix: Proof of formula (6). Formula (6) follows from the following string of identities and inequalities:

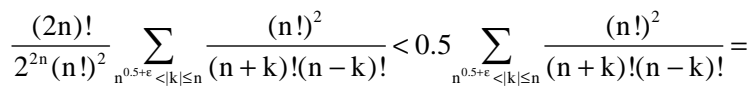

$$
\begin{aligned}
& 0.5 \sum_{n^{0.5+e_{e}}<|k| \leq n} \prod_{j=1}^{|k|} \frac{n-(k-j)}{n+j}=0.5 \sum_{n^{0.5+e_{e}}<|k| \leq n} \prod_{j=1}^{|k|}\left(1-\frac{k}{n+j}\right) \leq \\
& 0.5 \sum_{n^{0,5 i e}<|k| \leq n} \prod_{j=1}^{|k|}\left(1-\frac{k}{n+k}\right)=0.5 \sum_{n^{0,5+e}<<|k| n} \prod_{j=1}^{|k|}\left(\frac{n}{n+k}\right)^{|k|} \leq \\
& 0.5 \sum_{\mathrm{n}^{0.5+\varepsilon}<<|\mathrm{k}| \leq \mathrm{n}}\left(\frac{\mathrm{n}}{\mathrm{n}+\mathrm{n}^{0.5+\varepsilon}}\right) \mathrm{n}^{0.5+\varepsilon} \leq\left(\mathrm{n}-\mathrm{n}^{0.5+\varepsilon}\right)\left(\frac{\mathrm{n}}{\mathrm{n}+\mathrm{n}^{0.5+\varepsilon}}\right) \mathrm{n} 0^{.5+\varepsilon}= \\
& \frac{\mathrm{n}-\mathrm{n}^{0.5+\varepsilon}}{\left[\left(1+\frac{1}{\mathrm{n}^{0.5-\varepsilon}}\right) \mathrm{n}^{0.5-\varepsilon}\right] 2^{\mathrm{n \varepsilon}}} \leq \frac{\mathrm{n}-\mathrm{n}^{0.5+\varepsilon}}{2.5^{\mathrm{n}^{2 \varepsilon}}} \leq \frac{\mathrm{n}-\mathrm{n}^{0.5+\varepsilon}}{2.5^{\mathrm{n}^{2 \varepsilon}}} \leq 2^{-\mathrm{n}^{2 \varepsilon}}
\end{aligned}
$$

Proof of formula (7): Assume for simplicity's sake that $k \geq 0$. Applying a rather obvious inequality:

$$
\mathrm{e}^{\mathrm{x}-\mathrm{x}^{2}} \leq 1+\mathrm{x} \leq \mathrm{e}^{\mathrm{x}+\mathrm{x}^{2}}, \text { valid for }|\mathrm{x}| \ll 1
$$

with correspondingly $x=\frac{k-j}{n}$ and $x=\frac{j}{n}$ gives us:

$$
\begin{aligned}
& e^{-\frac{k-j}{n}-\left(\frac{k-j}{n}\right)^{2}} \leq 1-\frac{k-j}{n} \leq e^{-\frac{k-j}{n}+\left(\frac{k-j}{n}\right)^{2}}, \\
& e^{\frac{j}{n}-\left(\frac{j}{n}\right)^{2}} \leq 1+\frac{j}{n} \leq e^{\frac{j}{n}+\left(\frac{j}{n}\right)^{2}}
\end{aligned}
$$

Dividing the first one by the second yields:

$$
\mathrm{e}^{-\frac{k}{n}-\frac{(k-j)^{2}+j^{2}}{n^{2}}} \leq \frac{1-\frac{k-j}{n}}{1+\frac{j}{n}} \leq e^{-\frac{k}{n}+\frac{(k-j)^{2}+j^{2}}{n^{2}}}
$$

which, with the help of $\sum_{j=1}^{k}\left[(k-j)^{2}+j^{2}\right] \leq(k+1)^{3} \leq$ $\left(\mathrm{n}^{0.5+\varepsilon}+1\right)^{3} \leq 8 \mathrm{n}^{1.5+3 \varepsilon}$ gives us:

$$
\begin{aligned}
& e^{-\frac{k^{2}}{n}-8 n^{-0.5+3 e}} \leq e^{-\sum_{j=1}^{k}\left[\frac{k}{N}+\frac{(k-j)^{2}+j^{2}}{n^{2}}\right]} \leq \prod_{j=1}^{k} \frac{1-\frac{k-j}{n}}{1+\frac{j}{n}} \leq \\
& e^{\sum_{j=1}^{k}\left[-\frac{k}{N}+\frac{(k-j)^{2}+j^{2}}{n^{2}}\right]} \leq e^{-\frac{k^{2}}{n}+8 n^{-0.5+3 e}}
\end{aligned}
$$

Formula (7) follows.

\section{ACKNOWLEDGMENT}

The research was partially supported by the National Science and Engineering Research Council of Canada. 


\section{REFERENCES}

1. Brun, V., 1951. Walliss og Brounckers formler for $\pi$ (in Norwegian). Norsk Matematisk Tidskrift, 33: 73-81.

2. Mavromatis, H., 1992. The Wallis formula for $\pi$, including two new expressions involving irrationals. Int. J. Comput. Math., 43: 197-203. http://eprints.kfupm.edu.sa/8596/

3. Miller, S.J., 2008. A probabilistic proof of Wallis's formula for $\pi$. Am. Math. Monthly, 115: 740-745. http://adsabs.harvard.edu/abs/2007arXiv0709.2181 $\mathrm{M}$

4. Sondow, J., 2005. A faster product for $\pi$ and a new integral for $\ln \frac{\pi}{2}$. Am. Math. Monthly, 112: 729-734. http://www.jstor.org/stable/30037575
5. Wastlund, J., 2005. An elementary proof of Wallis product formula for $\pi$. Linkop. Stud. Math., 2: 1-5. http://www.ep.liu.se/ea/lsm/2005/002/lsm05002.pdf

6. Yaglom, A.M. and I.M. Yaglom, 1953. An elementary derivation of the formulas of Wallis, Leibnitz and Euler for the number $\pi$ (in Russian). Uspechi Matematiceskich Nauk, 8: 181-187. http://www.mathnet.ru/php/archive.phtml? wshow= paper\&jrnid=rm\&paperid=8256\&option_lang=eng 